\section{Shape Control in Sendzimir Mills Using Both Crown and Intermediate Roll Actuators}

\section{J. V. RINGWOOD AND M. J. GRIMBLE}

Abstract-The solution to the shape control problem for a Sendzimir mill, utilizing the As-U-Roll shape actuators, is well understood. The tapered first intermediate rolls, however, provide a more powerful shape control device. This note describes a control philosophy utilizing both the As-U-Rolls and first intermediate rolls as shape control mechanisms. A robustness result for the design is developed which is useful, since such a mill is normally used to roll a large number of materials, and a single controller must therefore be employed for many different schedules. Finally, a variety of simulation results are presented, showing the transient responses and performance of the multivariable shape control system.

\section{Introduction}

The shape control problem (the control of internal strip stress) in Sendzimir mills, utilizing the As-U-Roll (AUR) actuators, has been studied in some depth [1], [2] and indeed a shape control scheme, previously described [3], utilizing the AUR's is now approaching the final commissioning stages. Automatic shape control by means of the first intermediate rolls (FIR's) as a shape control device has, to date, received relatively little attention. Fig. 1 shows the location of both sets of actuators on the Sendzimir mill.

The first intermediate rolls may be moved in or out of the rolling cluster, and since they possess a taper (see Fig. 2), they can affect the roll bending profile in the mill and hence the shape profile [4]. Their primary function is to control shape at the strip edges, and it is the edge zones of the strip which are covered by the tapered part of the rolls. Due to their proximity to the strip, the FIR's provide a very powerful shape control device, and can produce high order bending in the workrolls. This is in contrast to the As-U-Rolls, whose bending effects are smoothed out by the stiffness of the intervening second intermediate and back-up rolls (see Fig. 1).

The relative importance of the FIR's as a shape control device is increased when consideration is given to the mechanical restrictions which inhibit certain profiles being set up on the AUR's. These limitations arise due to the danger of fracturing the back-up roll shaft by demanding extreme (and opposite) displacements in adjacent actuators. A certain amount of safety is achieved by limiting the AUR actuator profiles to fourth order (through parameterization [1]), but an analysis by Dutton [3] has shown this to be insufficient. The FIR's, on the other hand, do not suffer from such relative positional restrictions.

\section{The Sendzimir Mill Model}

The Sendzimir mill model has been adequately described elsewhere [3], [5], [6] and merely the form of the final (linearized) transfer function model is given here as

$$
y=g(s)\left[G_{a} \quad G_{i}\right] u
$$

where

$$
\begin{gathered}
y=\text { measured shape profile }\left(\in R^{8}\right) \\
u=\text { actuator inputs }\left(\in R^{10}\right) .
\end{gathered}
$$

Note that

$$
u=\left[\begin{array}{l}
u_{a} \\
u_{i}
\end{array}\right] \quad \begin{aligned}
& u_{a}=\mathrm{AUR} \text { inputs }\left(\in R^{s}\right) \\
& u_{i}=\text { FIR inputs }\left(\in R^{2}\right) .
\end{aligned}
$$

Manuscript received June 23, 1987; revised March 10, 1989. This work was supported by GEC Electrical Projects, Rugby and British Steel Corporation, Sheffield.

J. V. Ringwood is with the School of Electronic Engineering, Dublin City University, Glasnevin, Dublin, Ireland.

M. J. Grimble is with the Department of Electronic and Electrical Engineering, Industrial Control Unit, University of Strathclyde, Glasgow, United Kingdom.

IEEE Log Number 8933390

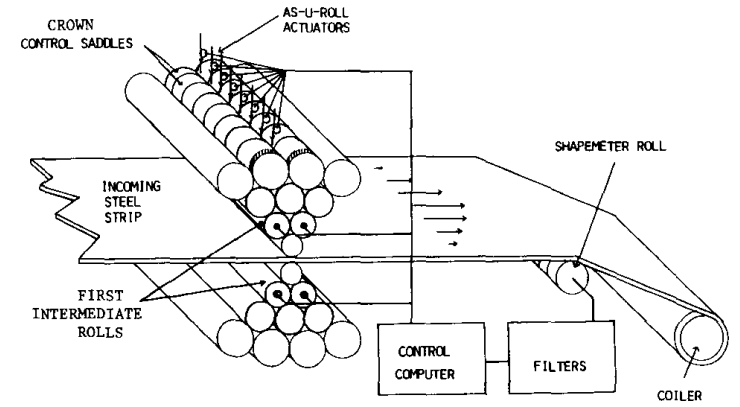

Fig. 1. Physical layout of Sendzimir mill.

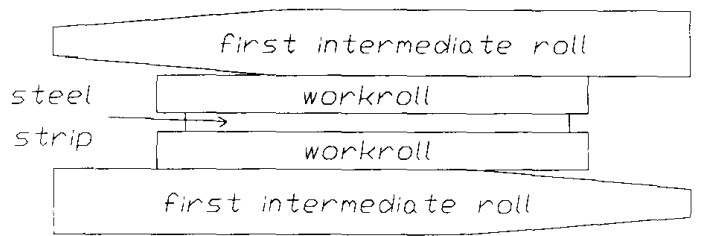

Fig. 2. First intermediate roll profile.

$G_{a}$ and $G_{i}$ are matrices of constant gains, relating roll-gap shape profile to actuator inputs (for AUR's and FIR's, respectively). The scalar transfer function $g(s)$ has the form

$$
g(s)=\frac{e^{-\tau_{1} s}}{(1+2.0 s)\left(1+\tau_{2} s\right)\left(1+\tau_{3} s\right)}
$$

where

$$
\tau_{1}=D / v, \quad \tau_{2}=D_{1} / v
$$

$D=$ distance from roll-gap to shapemeter $(2.91 \mathrm{~m})$

$D_{1}=$ distance from roll-gap to coiler $(5.32 \mathrm{~m})$

$v=$ strip velocity in $\mathrm{m} / \mathrm{s}$.

$\tau_{3}$ corresponds to a shapemeter time constant, which is varied for the different strip speeds as shown below

$$
\begin{array}{cccc}
\text { speed }(\mathrm{m} / \mathrm{s}) & 0 \rightarrow 2 & 2 \rightarrow 5 & 5 \rightarrow 15 \\
\tau_{3}(\mathrm{~s}) & 1.43 & 0.74 & 0.3
\end{array}
$$

\section{Shape Profile Parameterization}

Although the shapemeter output is modeled as an 8-point profile, the actual shapemeter produces a number of outputs ranging from 17 to 31 , depending on strip width. In order to provide a consistent number of outputs, a shape profile parameterization is used. Instead of controlling the actual shape or stress pattern, a number of parameters, or attributes, of the shape profile are controlled. Following a least-squares analysis based on a number of equally-spaced available measurements [3], [5], the best parameter fit is given by the Gram polynomials [7]. The first four Gram polynomials (excluding the zeroth order) are roughly linear, quadratic, cubic, and quartic in form, and it is the coefficients of these four parameters in the shape profile which will be controlled. Shape profiles corresponding to the higher order polynomials are not required to be controlled and more importantly, attempts to set up high-order roll bending in the mill can cause cracking of the back-up rolls. For this reason, a transformation is also used to limit the number of control inputs to the AUR actuators. This transformation is similar to that for the outputs, but in this case four control inputs are used to control eight actuators (hence limiting the bending to fourth order). A transformation, matrix corresponding to eight available measurements, evaluated from 


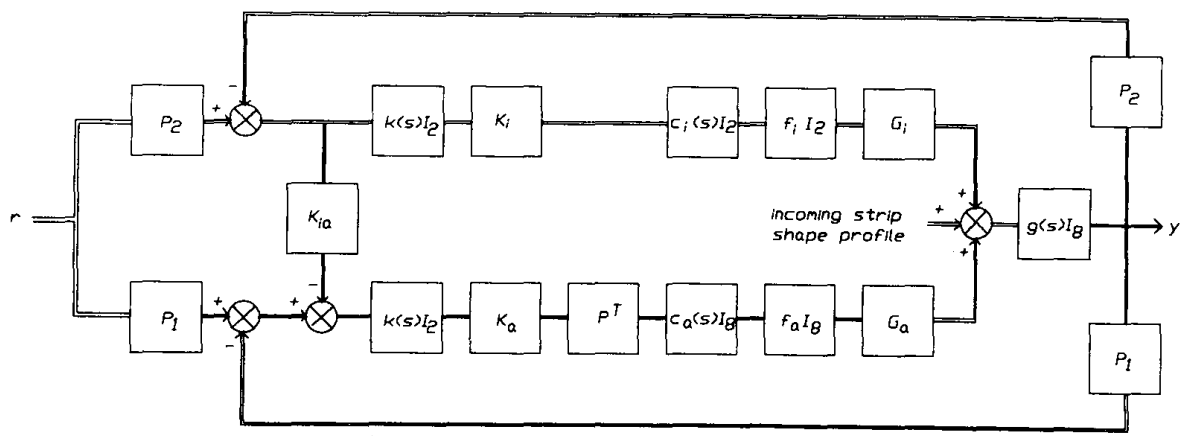

Fig. 3. Structure of multiloop control system.

the Fisher and Yeates tables [8] is

$P=\left[\begin{array}{rrrrrrrr}-0.54 & -0.38 & -0.23 & -0.08 & 0.08 & 0.23 & 0.38 & 0.54 \\ 0.54 & 0.08 & -0.23 & -0.38 & -0.38 & -0.23 & 0.08 & 0.54 \\ -0.43 & 0.31 & 0.43 & 0.18 & -0.18 & -0.43 & -0.31 & 0.43 \\ 0.28 & -0.53 & -0.12 & 0.36 & 0.36 & -0.12 & -0.53 & 0.28\end{array}\right]$

Note that a complete parameterization analysis is given in [1]. The linearized (and parameterized) mill TFM is given as

$$
G(s)=g(s) P\left[G_{a} P^{T} \quad G_{i}\right]
$$

\section{A Multiloop Control Solution}

In this approach, the FIR's are used to control different shape parameter coefficients than the AUR's, and a cross-coupling term used to alleviate interaction problems. The multiloop structure is shown in Fig. 3

\section{A. Design Approach}

In this approach, two parameter coefficients are controlled by each actuator set. It is not important at this stage how the parameters are allocated, the prime consideration being that the FIR and AUR parameterizations are mutually orthogonal. The Gram polynomials, mentioned in Section III, are used for shape profile parameterization. The matrix $P_{1}$ will be used to represent the AUR parameterization, and $P_{2}$ the FIR parameterization, where $P_{1}, P_{2} \in R^{2 \times 8}$.

The reasoning behind the multiloop structure is relatively straightforward. Since the FIR system has only two inputs, it can, at most, control only two shape parameter coefficients. The FIR loop, therefore, is chosen as the independent loop, and is diagonalized with respect to the parameter set $\boldsymbol{P}_{2}$, in the arrangement shown in Fig. 3, using $K_{i} \in R^{2 \times 2}$.

However, some undesirable shape components in the range space of $P_{1}$ are produced at the roll-gap by the FIR's, since they have no control over this parameter set. From knowledge of $G_{i}$, these components may be evaluated, and the parameter demand in the AUR loop adjusted accordingly via the cross-coupling term $K_{i a} \in R^{2 \times 2}$.

The AUR loop (dependent loop) can, unlike the FIR's, control all four parameter coefficients, since it has eight inputs (reduced to four by the parameterization). For the current configuration, however, only two parameter coefficients (corresponding to $P_{2}$ ) are required to be controlled. The demand in the parameter coefficients corresponding to $P_{2}$ are set to zero, therefore ensuring that no undesirable shape components in the range space of $P_{2}$ are produced at the roll-gap by the AUR's. The AUR controller, $K_{a}^{*} \in R^{4 \times 4}$ as a result, has four inputs, two of which are zero. An expression for the equivalent two-input/four-output controller, $K_{a} \in R^{4 \times 2}$ (shown in Fig. 3) is derived in Appendix I.

Theorem: The system is diagonalized by the choice of controllers $K_{i}$, $K_{a}^{*}$, and $K_{i a}$ as follows:

$$
K_{i}=\left(P_{2} G_{i}\right)^{-1} \quad \in R^{2 \times 2}
$$

$$
K_{a}^{*}=\left(P G_{a} P^{T}\right)^{-1} \quad \in R^{4 \times 4}
$$

where

$$
P=\left[\begin{array}{l}
P_{1} \\
P_{2}
\end{array}\right]
$$

$$
K_{i a}=P_{1} G_{i}\left(P_{2} G_{i}\right)^{-1} \in R^{2 \times 2} .
$$

Proof: From Appendix I,

$$
K_{a}=\left[\begin{array}{l}
\left(Q_{1}-Q_{2} Q_{4}^{-1} Q_{3}\right)^{-1} \\
\left(Q_{2}-Q_{1} Q_{3}^{-1} Q_{4}\right)^{-1}
\end{array}\right]
$$

where $Q_{1}, Q_{2}, Q_{3}$, and $Q_{4}$ may be identified from (A1.2).

Ignoring the plant dynamics (since the dynamics in each path are identical), the system forward path transfer function matrix may be written (from Fig. 3) as

$$
\begin{aligned}
& F=\left[\begin{array}{c}
P_{1} \\
P_{2}
\end{array}\right]\left[\begin{array}{ll}
G_{a} P^{T} & G_{i}
\end{array}\right]\left[\begin{array}{cc}
K_{a} & -K_{a} K_{i a} \\
0 & K_{i}
\end{array}\right] \\
& =\left[\begin{array}{ll}
P_{1} G_{a} P^{T} K_{a} & P_{1} G_{i} K_{i}-P_{1} G_{a} P^{T} K_{a} K_{i a} \\
P_{2} G_{a} P^{T} K_{a} & P_{2} G_{i} K_{i}-P_{2} G_{a} P^{T} K_{a} K_{i a}
\end{array}\right]
\end{aligned}
$$

where

$$
F \in R^{4 \times 4} \text {. }
$$

For the system to be diagonal, it is required that

$$
F=I_{4} \text {. }
$$

Equivalently, if $F$ is partitioned as

$$
F=\left[\begin{array}{ll}
F_{1} & F_{2} \\
F_{3} & F_{4}
\end{array}\right]
$$

with obvious identification of $F_{1}, F_{2}, F_{3}$, and $F_{4} \in R^{2 \times 2}$, then the required conditions on $F_{1}, F_{2}, F_{3}$, and $F_{4}$ are

$$
F_{1}=F_{4}=I_{2}, \quad F_{2}=F_{3}=0 \text {. }
$$

Examining each term in turn

i) For block $F_{1}$ : Using (9) and the identity in (A1.2), yields

$$
F_{1}=Q_{1}\left(Q_{1}-Q_{2} Q_{4}^{-1} Q_{3}\right)^{-1}+Q_{2}\left(Q_{2}-Q_{1} Q_{3}^{-1} Q_{4}\right)^{-1} .
$$

Applying the Householder inversion formula [9] to the term $\left(Q_{1}\right.$ $\left.Q_{2} Q_{4}^{-1} Q_{3}\right)^{-1}$ yields

$$
\begin{aligned}
& \left(Q_{1}-Q_{2} Q_{4}^{-1} Q_{3}\right)^{-1} \\
& =Q_{1}^{-1}+Q_{1}^{-1} Q_{2}\left(Q_{4}-Q_{3} Q_{1}^{-1} Q_{2}\right)^{-1} Q_{3} Q_{1}^{-1} \\
& =Q_{1}^{-1}-Q_{1}^{-1} Q_{2}\left(Q_{2}-Q_{1} Q_{3}^{-1} Q_{4}\right)^{-1}
\end{aligned}
$$




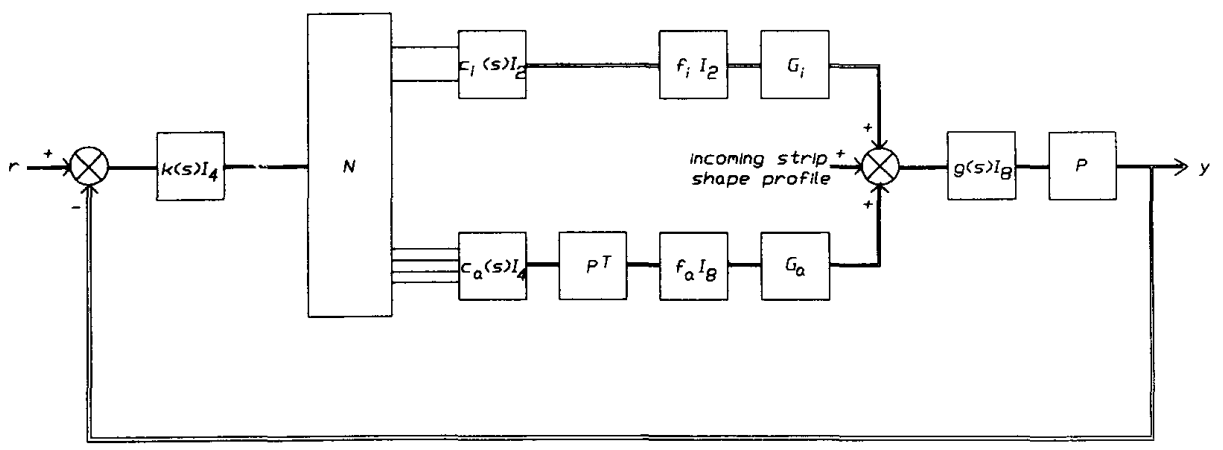

Fig. 4. Structure of unified control system.

Substituting back for $\left(Q_{1}-Q_{2} Q_{4}^{-1} Q_{3}\right)^{-1}$ in (13) gives

$$
\begin{aligned}
F_{1}= & Q_{1}\left[Q_{1}^{-1}-Q_{1}^{-1} Q_{2}\left(Q_{2}-Q_{1} Q_{3}^{-1} Q_{4}\right)^{-1}\right] \\
& +Q_{2}\left(Q_{2}-Q_{1} Q_{3}^{-1} Q_{4}\right)^{-1} \\
= & I_{2}-Q_{2}\left(Q_{2}-Q_{1} Q_{3}^{-1} Q_{4}\right)^{-1}+Q_{2}\left(Q_{2}-Q_{1} Q_{3}^{-1} Q_{4}\right)^{-1} \\
= & I_{2} .
\end{aligned}
$$

ii) For block $F_{3}$ :

$$
\begin{aligned}
F_{3} & =Q_{3}\left(Q_{1}-Q_{2} Q_{4}^{-1} Q_{3}\right)^{-1}+Q_{4}\left(Q_{2}-Q_{1} Q_{3}^{-1} Q_{4}\right)^{-1} \\
& =\left(Q_{1} Q_{3}^{-1}-Q_{2} Q_{4}^{-1}\right)^{-1}-\left(Q_{1} Q_{3}^{-1}-Q_{2} Q_{4}^{-1}\right)^{-1} \\
& =0 .
\end{aligned}
$$

iii) For block $F_{2}$ :

$$
F_{2}=P_{1} G_{i} K_{i}-P_{1} G_{a} P^{T} K_{a} K_{i a}
$$

It has already been shown in (15) that $P_{1} G_{a} P^{T} K_{a}=I_{2}$, giving

$$
F_{2}=P_{1} G_{i} K_{i}-K_{i a}
$$

Using (5) and (8) yields

$$
F_{2}=P_{1} G_{i}\left(P_{2} G_{i}\right)^{-1}-P_{1} G_{i}\left(P_{2} G_{i}\right)^{-1}
$$$$
=0 \text {. }
$$

iv) For block $F_{4}$ :

$$
F_{4}=P_{2} G_{i} K_{i}-P_{2} G_{a} P^{T} K_{a} K_{i a} .
$$

Using (16) and (5) gives

$$
F_{4}=P_{2} G_{i} K_{i}=P_{2} G_{i}\left(P_{2} G_{i}\right)^{-1}=I_{2}
$$

and it may be concluded that the system shown in Fig. 3 is diagonal for the controller choices of (12) to (15). The overall controller matrix is

$$
C 1=\left[\begin{array}{cc}
K_{a}^{*} & -K_{a}^{*} K_{i a} \\
0 & K_{i}
\end{array}\right] .
$$

The system has therefore been reduced to four identical SISO systems in parallel, each with forward path transfer function $g(s) k(s), k(s)$ being chosen to give suitable stability and dynamic performance characteristics.

\section{B. Parameter Allocation}

A number of combinations of parameters, which are to be controlled by each actuator set, exist. Although $6\left({ }^{4} C_{2}\right)$ combinations are possible, only one case will be examined here, one other case being documented in $[5]$.

Writing $P$, the transformation matrix given in Section IV, as

$$
\begin{gathered}
P^{T}=\left(\begin{array}{llll}
p_{1} & p_{2} & p_{3} & p_{4}
\end{array}\right) \\
\text { where } p_{1}, p_{2}, p_{3} \text { and } p_{4} \in R^{8}
\end{gathered}
$$

the case under consideration may be identified as

$$
P_{1}^{T}=\left(\begin{array}{ll}
p_{1} & p_{2}
\end{array}\right), \quad P_{2}^{T}=\left(\begin{array}{ll}
p_{3} & p_{4}
\end{array}\right)
$$

that is, the AUR's controlling the linear and quadratic shape parameter coefficients, and the FIR's controlling the cubic and quartic shape parameter coefficients. This choice of $P_{1}$ and $P_{2}$ accords with rolling practice (manual shape control), where the AUR's are used to control up to (and including) second order shape profiles, the FIR's being used to control shape at the strip edges (high order profiles). The reasoning behind this practice is that by setting up low order profiles on the AUR's, the restrictions regarding their relative movements are not violated, allowing their full potential to be realized. By using the FIR's to control the high order (edge) profiles, their best potential is realized, since their influence is greatest at the strip edges.

\section{A Unified Design Philosophy}

It will be shown in a forthcoming section that the design presented in Section IV has poor robustness properties to variations in the mill gain matrices. The following section presents an alternative diagonalization procedure with improved robustness properties.

\section{A. Design Approach}

The closed-loop structure which will be used in this design procedure is shown in Fig. 4. The linearized (and transformed) plant transfer function matrix excluding dynamics is given from (4) as

$$
G=P\left[G_{a} P^{T} \quad G_{i}\right] \in R^{4 \times 6} .
$$

Let a right inverse, $C 2 \in R^{6 \times 4}$, be defined such that

$$
\left[P G_{a} P^{T} \quad P G_{i}\right] C 2=I_{4} .
$$

Again, the system has been reduced to four SISO systems in parallel, each with forward path transfer function $g(s) k(s)$.

\section{B. Choice of a Right Inverse}

It can be shown [1] that $P G_{a} P^{T}\left(\in R^{4 \times 4}\right.$ ) is full rank, and hence that the matrix $G$ [given in (24)] is full row rank. Therefore, a right inverse, $C 2$, exists but is not necessarily unique [10]. The resulting design freedom may be exploited by minimizing the norm of the control inputs to the plant actuators. This helps to ensure that actuator wear is kept to a minimum and that the actuators do not attempt to violate their relative positional restrictions. The required right inverse which minimizes $u^{T} u$ is evaluated as

$$
C 2=G^{T}\left(G G^{T}\right)^{-1} .
$$

A proof, using Lagrange multipliers, is given in [10].

\section{Dynamic Precompensator Design}

$k(s)$ was designed for low, medium, and high speed plants using a combination of frequency response and simulation trials. The controller transfer function (for a single loop) is

$$
k(s)=\frac{k_{1}(1+2.0 s)}{(1+1000 s)(1+0.9 s)}
$$


where $k_{1}$ is given as

$$
\begin{array}{cccc}
\text { strip speed }(\mathrm{m} / \mathrm{s}) & 0 \rightarrow 2 & 2 \rightarrow 5 & 5 \rightarrow 15 \\
k_{1} & 100 & 200 & 500
\end{array}
$$

Note that a simple gain scheduling technique is used in $k(s)$ for changes in strip speed. A fixed strip speed is normally used for a complete run, the speed being determined by the quality and gauge of the strip and the amount of reduction required. Good steady-state response is ensured by placing a pole at $s=0.001$.

\section{Robustness Analysis}

Gauge reduction on the Sendzimir 20-roll Cold Rolling Mill is a multipass, multischedule process. For each steel coil rolled, a particular schedule is chosen according to strip width, initial gauge, final gauge, quality, and material of the coil. The schedule also specifies the number of passes the strip will undergo to achieve the required reduction in gauge. Since the percentage reduction varies with the pass number, and the hardness of the material increases as it is reduced, the mill matrices $G_{m}$ and $G_{i}$ are a function of pass number. It is not practical to store a precompensator matrix for each schedule and pass, and hence a smaller subset must be used. It may be possible to use one precompensator matrix for a number of passes, or indeed all the passes of a given schedule. However, it is important to have a measure of the allowable variations in the elements of $G_{m}$ and $G_{i}$ to see to what extent this simplification may be achieved while maintaining stability. It is also important to know the extent to which the modeling inaccuracies in the mill matrices will be tolerated.

For the current problem, an analysis based on variations in the elements of $G_{a}$ and $G_{i}$ is appropriate. The advantage of using element data is that the information on the system structure is retained in addition to the position and relative magnitudes of the errors. Furthermore, data on the errors in the elements of $G_{a}$ and $G_{i}$ are readily obtainable. This type of approach has recently been shown to be a useful and viable route to robust design in general [11], [12].

\section{A. Design Robustness with Respect to Errors in $G_{a}$ and $G_{i}$}

In the following analysis, it is assumed that a precompensator matrix $K(s) \in R(s)^{6 \times 4}$ has been designed for a nominal plant $G(s)$ but that $G_{a}$ and $G_{i}$ are subject to perturbations $\Delta_{a} \in R^{8 \times 8}$ and $\Delta_{i} \in R^{8 \times 2}$, respectively. Note that

$$
K(s)=K k(s)
$$

where $K \in R^{6 \times 4}$ is the diagonalizing controller (described in Sections IV and $\mathrm{V}$ ), and $k(s)$ the dynamic precompensator (described in Section VI). It is assumed that $k(s)$ stabilizes the plant dynamic transfer function $g(s)$.

Let a transformed perturbation matrix $\Delta$ be defined as

$$
\Delta=\left[P \Delta_{a} P^{T}, \quad P \Delta_{i}\right] .
$$

The stability of the feedback system is described by the return difference

$$
\begin{aligned}
\mid I_{4} & +g(s) k(s) P\left[\left(G_{a}+\Delta_{a}\right) P^{T},\left(G_{i}+\Delta_{i}\right)\right] K \mid \\
& =\left|I_{4}+g(s) k(s) P\left[G_{a} P^{T}, G_{i}\right] K+g(s) k(s) P\left[\Delta_{a} P^{T}, \Delta_{i}\right] K\right| \\
& =|A||B|
\end{aligned}
$$

where

$$
\begin{gathered}
A=I_{4}+g(s) k(s) P\left[G_{a} P^{T}, G_{i}\right] K \\
B=I_{4}+\left\{g(s) k(s) P\left[\Delta_{a} P^{T}, \Delta_{i}\right] K\right\}^{-1} g(s) k(s) P\left[G_{a} P^{T}, G_{i}\right] K .
\end{gathered}
$$

Clearly, system stability is determined by the ' $B$ ' determinant, since the ' $A$ ' determinant is merely the return difference of the unperturbed system. By noting that

$$
\left[P G_{a} P^{T} \quad P G_{i}\right] K=I_{4}
$$

which is true for both the multilevel and the right-inverse controllers, the stability of the perturbed system is determined by the condition

$$
\left|I_{4}+(1+g k)^{-1} g k \Delta K\right| \neq 0 \quad \text { for } \operatorname{Re}(s) \geq 0
$$

substituting from (29) and dropping the $s$-dependence for clarity. The condition in (32) may be replaced by the more conservative condition

$$
1>\sum_{j=1}^{4}\left|F_{r j}(s)\right| \quad 1 \leq r \leq 4
$$

where $F_{r j}$ are the elements of the matrix $F(s) \in R(s)^{4 \times 4}$, given by

$$
F(s)=(1+g k)^{-1} g k \Delta K .
$$

Since $F(s)$ is strictly proper and analytic and bounded in the interior of $D$, the suprema are achieved on the imaginary axis, and the frequency dependent condition of (33) may be replaced by the frequency independent condition

$$
1>\sum_{j=1}^{4} \sup _{\omega \geq 0}\left|F_{r j}(j \omega)\right| \quad 1 \leq r \leq 4 .
$$

Defining the maximum value of the closed-loop frequency response as

$$
\gamma=\sup _{\omega \geq 0}\left|(1+g k)^{-1} g k\right|
$$

the condition expressed in (35) becomes

$$
1>\sum_{j=1}^{4}\left|\gamma(\Delta K)_{r j}\right| \quad 1 \leq r \leq 4 .
$$

Given the perturbation $\Delta$ the controller matrix $K$ and $\gamma$ (from a Nicholl's Chart), it is possible to determine the stability of the perturbed system by examining the inequalities given in (37). For the two cases under consideration, the controller matrix $K$ becomes

$$
K=C 1
$$

where $C 1$ is given in (22) for the multilevel controller, and

$$
K=C 2
$$

where $C 2$ is given in (26) for the right inverse controller.

\section{B. Mismatched Controller Evaluation}

An example is taken here, whereby a diagonalizing controller matrix $K$, calculated for Schedule $X$ Pass 1 , is to be used with a plant corresponding to Schedule $Y$ Pass 1 . For this case the variation in mill matrix element values was as much as 100 percent. It is required to evaluate the inequalities of (37) for this case to determine if stability is retained. No intermediate numerical results are provided but the calculation route is as follows.

i) Evaluate $\Delta_{a}$ and $\Delta_{i}$ from the mill matrices pertaining to the different schedules.

ii) Evaluate the transformed perturbation matrix $\Delta$.

iii) Calculate $\gamma$, the maximum value of the closed-loop frequency response from a Nicholl's Plot.

iv) Evaluate the product $\Delta K$ and multiply by $\gamma$ to give the inequality coefficients.

v) Sum the resulting coefficients over the rows and test for stability.

The resulting inequalities, calculated for both controllers $C 1$ and $C 2$ are as follows:

$$
\begin{array}{ll}
\text { Controller } C 1: & \text { Controller } C 2: \\
1>1.3911 & 1>0.2215 \\
1>2.3710 & 1>0.8352 \\
1>1.3539 & 1>0.1059 \\
1>1.5461 & 1>0.2591
\end{array}
$$




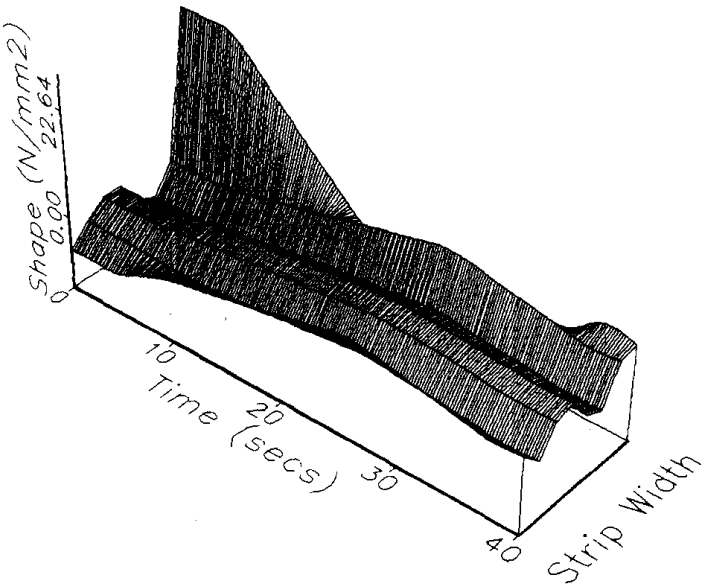

Fig. 5. Shape profile variations for multiloop system.

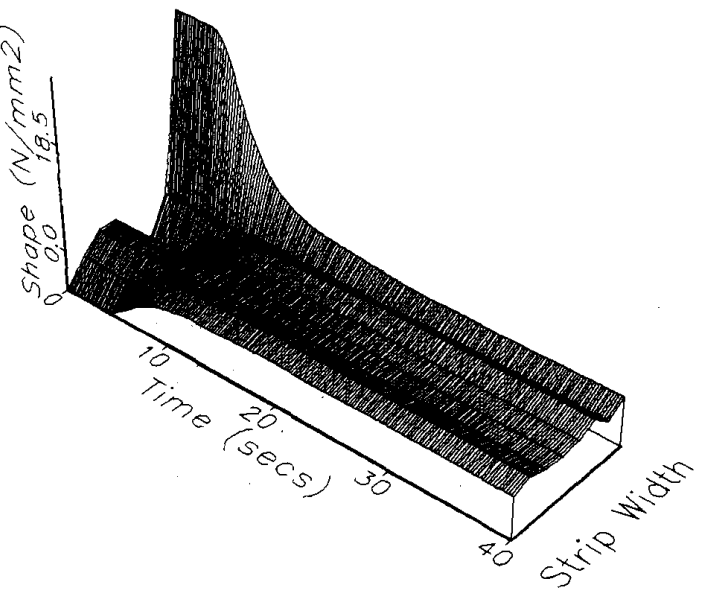

Fig. 6. Shape profile variations for unified system.

The above inequalities were evaluated using a value of $\gamma=0.99$ (obtained from a Nicholl's Chart), pertaining to a medium speed plant. Simulation results for controller $C 2$ in this mismatched case are given in Section VIII. It is seen that, for controller $C 2$, the inequality set is satisfied, indicating that stability is retained. For $C 1$, although stability is retained (confirmed by simulation tests [5]), the inequality set has not been satisfied. This is due to the conservatism built into the analysis via (33). In such cases, system stability (or rather instability) must be confirmed by simulation tests. Note, however, that when the inequalities are satisfied, stability is guaranteed.

\section{ViII. Performance Evaluation}

Nonlinear simulation tests were used to assess the performance of the shape control schemes developed in the preceding sections. The output shape profile variations with time for controller $C 1$ and $C 2$ are shown in Figs. 5 and 6, respectively. Note that control is applied after the simulation has been allowed to run for $3 \mathrm{~s}$. Both $C 1$ and $C 2$ have good steady-state performance (the residual profiles consisting of high order shape components) but the transient response of $C 2$ is superior.

As an example, Fig. 7 shows the parameter variations with time for $C 2$. As a comparative performance measure, Fig. 8 shows the sum of the magnitudes of the AUR and FIR inputs and the sum of the magnitudes of the shape errors across the strip width for both inverse (inv) and mulfiloop $(\mathrm{m} / \mathrm{l})$ compensators. The most noteworthy characteristic of this diagram is the relatively large input vector to the FIR's causing them to saturate, the integral effect in the dynamic precompensator producing the

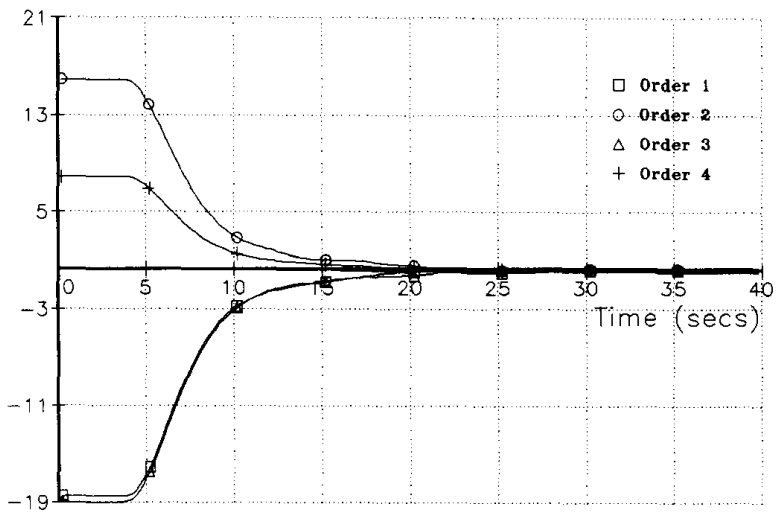

Fig. 7. Shape parameter variations for unified system.

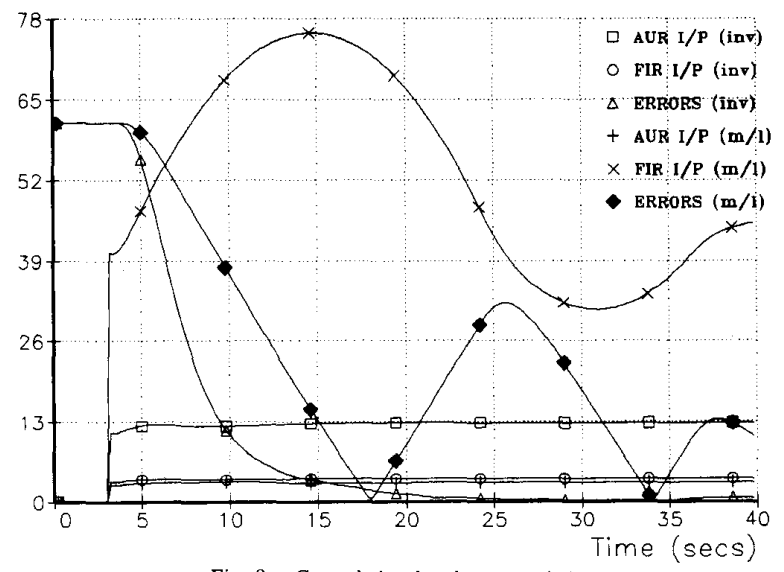

Fig. 8. Control signal and error variations.

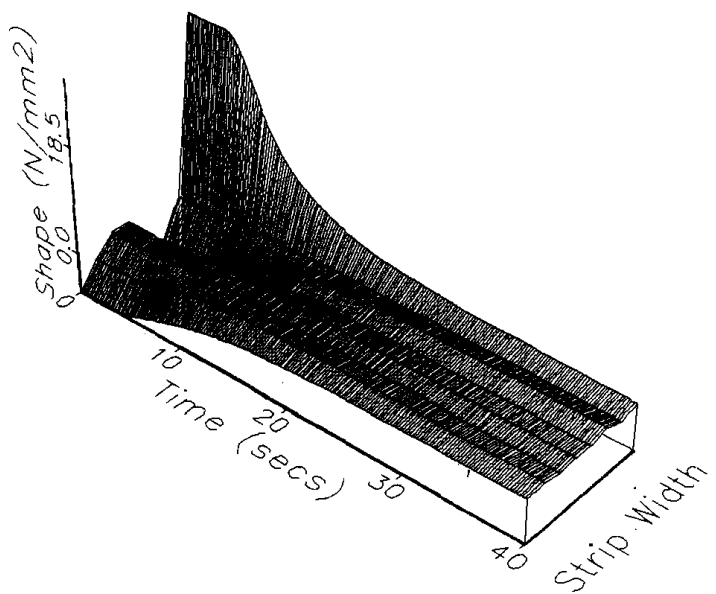

Fig. 9. Performance of unified system for mismatched compensator.

resulting overshoot. A better proportion between FIR and AUR inputs is achieved in the case of $C 2$ with the resulting smooth error profile.

Fig. 9 shows the shape profile variations for a mismatched $C 2$ controller for the case given in Section VII-B. This verifies that no instability is present, as predicted by the set of inequalities.

\section{Discussion}

Two controller designs have been examined, one of which minimizes the control inputs to the plant. The consequences of this minimization 
TABLE I

\begin{tabular}{cc}
\hline CONTROLLER & $\|K\|_{E}$ \\
\hline$C 1$ & 0.941 \\
$C 2$ & 0.183 \\
$C 3$ & 4.423
\end{tabular}

will now be examined more fully. This special feature of $C 2$ results in the elements of the $C 2$ matrix being small in magnitude compared to $C 1$. A quantitative measure of the magnitude of the matrix elements is given as the Euclidean norm [13], which is defined as

$$
\|K\|_{E}=\left[\sum_{i=1}^{6} \sum_{j=1}^{4} k_{i j}^{2}\right]^{1 / 2}
$$

where $k_{i j}$ are the elements of the given controller matrix. Calculation of the Euclidean norm for $C 1$ and $C 2$ gives the results in Table I. Note the inclusion of a norm value corresponding to a controller " $\mathrm{C3}$." $\mathrm{C3}$ corresponds to a multiloop controller, where the AUR's are used to control second- and fourth-order shape profiles (even orders) and the FIR's are used to control first- and third-order profiles (odd orders). This controller is documented fully in [5]. Controller $C 3$ is included in this section to allow a more complete controller comparison to be made.

From (37), the robustness of a particular controller is seen to depend on the magnitude of the elements of the controller matrix. Some conclusions, therefore, regarding the relative robustness of the various controllers may be made with respect to Table I. Controller $C 2$ appears to be the most robust, with $C 1$ and $C 3$ being progressively less robust. This is significant, since $C 1$ and $C 3$ both contain zero blocks [see (22)], and hence one would expect the matrix norms to be small.

If the efficiency of a controller is defined as that which minimizes control effort, then some conclusions regarding the efficiency of the different controller structures may also be deduced from Table I. In controller $C 2$, all four parameters may be set up on both the AUR's and FIR's. The relative distribution of the parameters on each actuator set is determined in an "optimal" sense so that the control input norms are minimized. In $C 1$ and $C 3$ the parameter allocation is fixed initially and the resulting matrix norms are large. The exceptionally large norm for $C 3$ indicates the difficulty of setting up first- and third-order profiles on the FIR's and second- and fourth-order profiles on the AUR's. It may therefore be concluded that this structure is inefficient (validated by simulation results given in [5]).

\section{Conclusions}

A variety of designs for the shape control of a Sendzimir mill utilizing both AUR and FIR actuators have been developed. The different designs allow different combinations of shape parameters to be set up on the different actuator sets. While the right inverse controller of Section $\mathrm{V}$ was shown to have the best performance and robustness properties, a multiloop structure may be more appropriate from mechanical or operator considerations (recalling that the configuration of Section IV-B corresponds with manual rolling practice).

Finally, the robustness of the control philosophies developed was expressed in terms of a series of strict linear inequalities. These inequalities are easily calculated from the elemental data available via the static model of Gunawardene [6]. Although the stability prediction of the analysis is sometimes conservative, satisfaction of the inequalities guarantees stability.

It is envisaged that a singular value decomposition could also have been used to diagonalize the constant plant TFM. However, such a decomposition would not have the same physical significance as the parameterization presented in Section III, where the shape profile is parameterized in terms of the natural bending modes present in the mill [2].

APPENDIX I

Here, an expression for $K_{a}$ in terms of $K_{a}^{*}$ is derived.
Let

$$
z=K_{a}^{*}\left(\begin{array}{c}
x_{1} \\
x_{2} \\
0 \\
0
\end{array}\right), \quad z \in R^{4}
$$

where $x_{1}$ and $x_{2}$ (scalars) are the inputs to the $K_{a}^{*}$ block.

$$
z=\left(P G_{a} P^{T}\right)^{-1}\left(\begin{array}{c}
x_{1} \\
x_{2} \\
0 \\
0
\end{array}\right)
$$

Now, $\left(P G_{a} P^{T}\right)^{-1}$ may be written as

$$
\left(\begin{array}{cc}
P_{1} G_{a} P_{1}^{T} & P_{1} G_{a} P_{2}^{T} \\
P_{2} G_{a} P_{1}^{T} & P_{2} G_{a} P_{2}^{T}
\end{array}\right)^{-1}=\left(\begin{array}{ll}
Q_{1} & Q_{2} \\
Q_{3} & Q_{4}
\end{array}\right)^{-1}
$$

with the obvious identification of $Q_{1}, Q_{2}, Q_{3}, Q_{4} \in R^{2 \times 2}$ and assuming that $Q_{1}$ to $Q_{4}$ are all square and invertible

$$
\left(\begin{array}{ll}
Q_{1} & Q_{2} \\
Q_{3} & Q_{4}
\end{array}\right)^{-1}=\left(\begin{array}{ll}
\left(Q_{1}-Q_{2} Q_{4}^{-1} Q_{e}\right)^{-1} & \left(Q_{3}-Q_{4} Q_{2}^{-1} Q_{1}\right)^{-1} \\
\left(Q_{2}-Q_{1} Q_{3}^{-1} Q_{4}\right)^{-1} & \left(Q_{4}-Q_{3} Q_{1}^{-1} Q_{2}\right)^{-1}
\end{array}\right)
$$

Proof given in Appendix II. From (A1.3)

$$
\begin{aligned}
z & =\left(\begin{array}{ll}
\left(Q_{1}-Q_{2} Q_{4}^{-1} Q_{3}\right)^{-1} & \left(Q_{3}-Q_{4} Q_{2}^{-1} Q_{1}\right)^{-1} \\
\left(Q_{2}-Q_{1} Q_{3}^{-1} Q_{4}\right)^{-1} & \left(Q_{4}-Q_{3} Q_{1}^{-1} Q_{2}\right)^{-1}
\end{array}\right)\left[\begin{array}{c}
x_{1} \\
x_{2} \\
0 \\
0
\end{array}\right] \\
& =\left[\begin{array}{l}
\left(Q_{1}-Q_{2} Q_{4}^{-1} Q_{3}\right)^{-1} \\
\left(Q_{2}-Q_{1} Q_{3}^{-1} Q_{4}\right)^{-1}
\end{array}\right]\left[\begin{array}{l}
x_{1} \\
x_{2}
\end{array}\right]
\end{aligned}
$$

The required transfer function matrix $K_{a}$ may now be identified as

$$
K_{a}=\left[\begin{array}{l}
\left(Q_{1}-Q_{2} Q_{4}^{-1} Q_{3}\right)^{-1} \\
\left(Q_{2}-Q_{1} Q_{3}^{-1} Q_{4}\right)^{-1}
\end{array}\right] .
$$

APPENDIX II

Inverse of Partitioned Matrix (Square Partitions)

Let

$$
\left(\begin{array}{ll}
Q_{1} & Q_{2} \\
Q_{3} & Q_{4}
\end{array}\right)^{-1}=\left(\begin{array}{ll}
R_{1} & R_{2} \\
R_{3} & R_{4}
\end{array}\right) .
$$

The matrices $Q_{1}, Q_{2}, Q_{3}$, and $Q_{4}$ are all assumed square and invertible The above equation may be rearranged to give

$$
\left(\begin{array}{ll}
Q_{1} & Q_{2} \\
Q_{3} & Q_{4}
\end{array}\right)\left(\begin{array}{ll}
R_{1} & R_{2} \\
R_{3} & R_{4}
\end{array}\right)=\left(\begin{array}{ll}
I & 0 \\
0 & I
\end{array}\right)
$$

whence

$$
\begin{aligned}
& Q_{1} R_{1}+Q_{2} R_{3}=I \\
& Q_{1} R_{2}+Q_{2} R_{4}=0 \\
& Q_{3} R_{1}+Q_{4} R_{3}=0 \\
& Q_{3} R_{2}+Q_{4} R_{4}=I .
\end{aligned}
$$


From (A2.1)

$$
R_{1}=Q_{1}^{-1}\left(I-Q_{2} R_{3}\right)
$$

and, from (A2.3)

$$
R_{1}=-Q_{3}^{-1} Q_{4} R_{3}
$$

giving

$$
Q_{1}^{-1}\left(I-Q_{2} R_{3}\right)=-Q_{3}^{-1} Q_{4} R_{3}
$$

or

$$
R_{3}=\left(Q_{2}-Q_{1} Q_{3}^{-1} Q_{4}\right)^{-1} .
$$

Similarly, using (A2.3) and (A2.3)

$$
-Q_{1}^{-1} Q_{2} R_{4}=Q_{3}^{-1}\left(I-Q_{4} R_{4}\right)
$$

giving

$$
R_{4}=\left(Q_{4}-Q_{3} Q_{1}^{-1} Q_{2}\right)^{-1} .
$$

Again, using (A2.1) and (A2.3) gives

$$
-Q_{4}^{-1} Q_{3} R_{1}=Q_{2}^{-1}\left(I-Q_{1} R_{1}\right)
$$

giving

$$
R_{1}=\left(Q_{1}-Q_{2} Q_{4}^{-1} Q_{3}\right)^{-1} .
$$

Finally, using (A2.2) and (A2.4) gives

$$
Q_{4}^{-1}\left(I-Q_{3} R_{2}\right)=-Q_{2}^{-1} Q_{1} R_{2}
$$

hence

$$
R_{2}=\left(Q_{3}-Q_{4} Q_{2}^{-1} Q_{1}\right)^{-1} .
$$

The above results may be combined to give

$$
\begin{aligned}
\left(\begin{array}{cc}
R_{1} & R_{2} \\
R_{3} & R_{4}
\end{array}\right) & =\left(\begin{array}{cc}
\left(Q_{1}-Q_{2} Q_{4}^{-1} Q_{3}\right)^{-1} & \left(Q_{3}-Q_{4} Q_{2}^{-1} Q_{1}\right)^{-1} \\
\left(Q_{2}-Q_{1} Q_{3}^{-1} Q_{4}\right)^{-1} & \left(Q_{4}-Q_{3} Q_{1}^{-1} Q_{2}\right)^{-1}
\end{array}\right) \\
& =\left(\begin{array}{ll}
Q_{1} & Q_{2} \\
Q_{3} & Q_{4}
\end{array}\right)^{-1} .
\end{aligned}
$$

ACKnowledgment

The authors are grateful for the contributions to the project by A. Kidd of GEC and A. Bill of BSC. Thanks are also due to Dr. K. Dutton of Sheffield City Polytechnic, Dr. M. A. Johnson and Dr. J. F. Barrett of the Industrial Control Unit, University of Strathclyde, and Prof. D. H. Owens of the Department of Mathematics, University of Strathclyde for their many helpful comments.

\section{REFERENCES}

[1] M. J. Grimble and J. Fotakis, "The design of shape control systems for Sendzimir mills," IEEE Trans. Automat. Contr., vol. AC-27, pp. 656-666, 1982.

[2] J. V. Ringwood, D. H. Owens, and M. J. Grimble, "Feedback design of a canonical multivariable system with application to shape control in Sendzimir mills," Proc. ACC, San Diego, CA, May 1990

[3] K. Dutton, "An investigation into the design and performance of a shape control system for a Sendzimir mill," Ph.D. dissertation, Sheffield City Polytechnic, 1983.

[4] C. A. Bravington, D. C. Barry, and C. H. McClure, "Design and development of a shape control system," in Proc. Metals Soc. Conf. on Shape Contr., Chester, Mar. 1976, pp. 82-88.

[5] J. V. Ringwood, "The design of shape control systems for a Sendzimir mill," Ph.D. dissertation, Univ. Strathclyde, 1984.

[6] G. W. D. M. Gunawardene, "Static model development for the Sendzimir cold rolling mill," Ph.D. dissertation, Sheffield City Polytechnic, 1982.

[7] J. V. Beck and K. J. Arnold, Parameter Estimation in Engineering and Science. New York: Wiley.
[8] R. A. Fisher and F. Yeates, Statistical Tables for Biological, Agricultural and Medical Research. Oliver and Boyd, 1925.

[9] A. S. Householder, Principles of Numerical Analysis. New York: McGrawHill, 1953.

[10] B. Noble, Applied Linear Algebra. Englewood Cliffs, NJ: Prentice-Hall, 1969.

[11] D. H. Owens and A. Chotai, "Robust control of linear dynamic systems using approximate models," Proc. IEE Part D, vol. 130, pp. 45-57, 1983.

[12] J. Lunze, "Multidimensional bounds for robust stability studies," Syst. Contr. Lett., vol. 4, pp. 85-89, 1984.

[13] W. Ledermann, Handbook of Applicable Mathematics Vol. 3-Numerical Methods. New York: Wiley, 1981.

\section{Optimal Intensity Control of a Queueing System with State-Dependent Capacity Limit}

HONG CHEN AND DAVID D. YAO

\begin{abstract}
We study a general single-stage queueing system, in which the input and output processes are modeled as point processes with stochastic intensities. The problem is to control both the input and the output intensities, subject to some state-dependent capacity limits, and the objective is to maximize a discounted value function. With reasonable assumptions on the capacity limits, we show there exists an optimal control that is of threshold type, characterized by a finite upper barrier (the lower barrier being zero). The results developed here provide theoretical justification for the optimality of the threshold control, which is widely applied in practice.
\end{abstract}

I. INTRODUCTION

We study a general single-stage queueing system, in which the input (arrival) and output (service completion) processes are modeled as point processes with stochastic intensities [1]. The problem is to control both the input and the output intensities, subject to some state-dependent capacity limits, and the objective is to maximize a discounted value function, with the "value" being revenue minus operating and holding costs.

As a motivating example, suppose the system is a production cell. Let the state of the system be the work-in-process inventory (i.e., the jobs that are present in the cell). The output process models the production of the cell, while the input process models the production of an upstream stage that feeds semiproducts into the cell; both processes are subject to control. There are also constraints to be met. For instance, since the production capacity of the cell is usually a function of the work-in-process inventory, given a work-in-process level there is a limit on the production rate of the cell. Given a work-in-process level, there is also a limit on the production rate of the upstream stage, in order to avoid overstock in the cell, for instance. These two sets of limits will be referred to as input and output capacity limits, respectively.

In practice, the operation of such a system is often governed by a threshold type of control, which is characterized by two threshold values: a lower barrier and an upper barrier. Whenever the work-in-process level reaches the upper barrier (signaling overstock), the input process is turned off (i.e., the production of the upstream stage is temporarily suspended); whenever the work-in-process level reaches the lower barrier (signaling understock), the output process is turned off (i.e., the production of the cell itself is temporarily suspended). If the work-inprocess level is in between the lower and the upper barriers, then both the input and the output processes run at the maximum allowed capacity limits. There are obvious advantages in exercising this type of thresh-

Manuscript received May 23, 1988; revised January 3, 1989. Paper recommended by Associate Editor, S. Verdu. This work was supported in part by NSF under Grant ECS 8658157 and by ONR under Contract N00014-84-K-0465. This work was completed while the authors were with the Division of Applied Sciences, Harvard University, Cambridge, MA 02138 .

H. Chen is with the Faculty of Commerce, University of British Columbia, Vancouver, B.C., V6T 148, Canada.

D. D. Yao is with the Department of Industrial Engineering and Operations Research, Columbia University, New York, NY 10027-6699.

IEEE Log Number 893328 